Obere Extremität 2020 · 15:273-283 https://doi.org/10.1007/s11678-020-00604-x Eingegangen: 17. Juni 2020

Angenommen: 2 . September 2020

Online publiziert: 7. Oktober 2020

(c) Der/die Autor(en) 2020

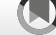

Check for
updates

Die Osteochondrosis dissecans (OD) ist eine Erkrankung, bei der fokale Veränderungen des subchondralen Knochens entstehen. Diese kommen v. a. an konvexen Gelenkanteilen vor. Das Ellenbogengelenk stellt nach dem Kniegelenk den zweithäufigsten Manifestationsort dar. Um konsekutiv anhaltende Beschwerden wie Funktionseinschränkungen oder Instabilitäten zu verhindern, bedarf es einer optimalen stadiengerechten Therapie. Ziel dieses Artikels ist es, eine aktuelle Übersicht über Erkenntnisse und Behandlungsempfehlungen der OD am Ellenbogengelenk darzustellen.

\section{Einleitung, Definition und Ätiologie}

Franz König beschrieb 1888 in seinem Werk „Über freie Körper im Gelenk“ eine entzündliche Erkrankung, bei der es fokal zu einer Veränderung des subchondralen Knochens sowie des überliegenden Knorpels kommt. Da er eine entzündliche Genese vermutete, nannte er sie „Osteochondritis dissecans“. Dieser Begriff findet heutzutage weiterhin Verwendung, selbst wenn histopathologische Studien einen entzündlichen Prozess bei der OD ausschließen. Die vielleicht treffendste Bezeichnung ist die Einordnung der OD in die Gruppe der osteochondralen Läsionen.

Über die genaue Ätiologie besteht weiterhin ein wissenschaftlicher Diskurs. Die Erklärungsansätze reichen von traumatischen, ischämischen, genetisch-prädisponierenden bis hin zu Osteosynthesestörungen.

Das signifikant vermehrte Vorkommen der OD am Ellenbogen bei Über-

\title{
Osteochondrosis dissecans des Ellenbogengelenks
}

kopfsportler stützt den traumatischen Erklärungsansatz als Folge repetitiver Mikrotraumen [1, 4, 10, 47]. Biomechanische Studien konnten aufzeigen, dass gerade der am häufigsten betroffene laterale Teil des Capitulums im Vergleich zum medialen Anteil des Capitulums weicher ist. Zudem ist der Radiuskopf im Verhältnis von härterer Beschaffenheit [44]. Repetitiver Valgusstress könnte daher im lateralen Anteil des Capitulums Mikrofrakturen verursachen und so eine OD begünstigen.

Der ischämische Erklärungsansatz wird z.B. durch die Forschung von Haraldsson [18] gestützt. Er zeigte auf, dass bei Heranwachsenden der Wachstumskern des lateralen Capitulums nur durch maximal zwei funktionelle Endarterien versorgt wird. Die reduzierte vaskuläre Versorgung ohne Kollateralkreisläufe begünstigt die OD am lateralen Capitulum zusätzlich. In der Literatur finden sich zudem auch andere ischämische Erklärungsansätze wie Infarkte oder Embolien als Ursache der stattfindenden Knochennekrose.

Familiäre Häufungen aber auch die Tatsache, dass die OD sich bilateral und polyartikulär ausprägen kann, legen die Theorie der genetischen Prädisposition nah [34]. Gleichsam finden sich Arbeiten, die genetische Erkrankungen wie der multiplen epiphysialen Dysplasie als prädisponierende Morbidität für eine OD postulieren [53]. Bisweilen fehlen auch hier eindeutige Evidenzen.

\section{》) Die Ätiologie der OD ist nicht umfassend erklärt}

Die OD am Ellenbogen ist eine seltene Erkrankung. Ihre Inzidenz wird mit
15-30 auf 100.000 Einwohner angegeben und unterliegt großen regionalen Schwankungen. Als zweithäufigstes betroffenes Gelenk entspricht die OD am Ellenbogen etwa $6 \%$ aller osteochondralen Läsionen [50]. Männer sind mit einem Verhältnis von 3:1 betont in der zweiten Lebensdekade signifikant häufiger betroffen [31]. Auch wenn die OD von direkt traumatisch-bedingten osteochondralen Läsionen im Kontext von Makrotraumen wie Ellenbogenfrakturen etc. differenziert werden muss, so finden sich bei fast jedem zweiten Patienten Assoziationen zu stattgehabten Traumata. Untersuchungen an erwachsenen Baseballspielern, wie die von Kida et al. [23], postulieren Prävalenzen von $3,4 \%$ der untersuchten Population. Neben Baseball sind auch Sportarten wie Gymnastik bzw. Kunsturnen häufiger mit osteochondralen Läsionen vergesellschaftet. Die überdurchschnittliche Belastung und repetitiver Valgusstress auf das Ellenbogengelenk während der Ausübung dieser Sportart sind erklärend für die höhere Inzidenz in diesem Kollektiv.

Differentialdiagnostisch muss die OD von den juvenilen avaskulären Nekrosen unterschieden werden. Der Morbus Panner, eine der häufigsten Nekrosen des Capitulum humeri und der Morbus Hegemann, eine seltenere Form der $\mathrm{Ne}$ krose mit Manifestation an der Trochlea humeri, sind klinisch von einer OD am Ellenbogen nicht zu unterscheiden. Nebst des jüngeren Erkrankungsalter (Morbus Panner 8-11 Jahre, Morbus Hegemann 11-13 Jahre) sind bei den juvenilen avaskulären Osteonekrosen der gesamte Gelenkabschnitt betroffen, z.B. das gesamte Capitulum bei Morbus Panner. Wie bereits beschrieben tritt 
Tab. 1 Klassifikationssysteme der Osteochondrosis dissecans für bildgebende Verfahren als auch intraoperative Befunde (röntgenbasierte Einteilung nach Minami et al. [50])

\begin{tabular}{l|l}
\hline Graduierung & Beschreibung \\
\hline Grad I & $\begin{array}{l}\text { Stabile Läsion mit Zysten im Capitulum } \\
\text { Klare Separierung zwischen der Osteochondrosis dissecans und dem sub- } \\
\text { chondralen Knochen }\end{array}$ \\
\hline Grad III & Freier Gelenkkörper
\end{tabular}

Tab. 2 Klassifikationssysteme der Osteochondrosis dissecans für bildgebende Verfahren als auch intraoperative Befunde (MRT-basierte Klassifikation nach Dipaola et al. [16])

\begin{tabular}{|c|c|c|}
\hline Graduierung & Evaluation & Beschreibung \\
\hline Grad I & Frühstadium & Verdickung des Knorpels ohne Kontinuitätsunterbrechung \\
\hline Grad lla & Stabil & $\begin{array}{l}\text { Initiale Separierung des Knorpels mit hypointensem Signal } \\
\text { (T2) subfragmentär (fibröse Verbindung) }\end{array}$ \\
\hline Grad llb & Instabil & Inkomplette Ablösung des osteochondralen Fragments \\
\hline Grad III & Instabil & $\begin{array}{l}\text { Komplette Ablösung des Fragments ohne Dislokation, } \\
\text { hyperintenses Signal (T2) subfragmentär (freies Fragment) }\end{array}$ \\
\hline Grad IV & Finales Stadium & Komplette Ablösung des Fragments mit Dislokation \\
\hline
\end{tabular}

Tab. 3 Klassifikationssysteme der Osteochondrosis dissecans für bildgebende Verfahren als auch intraoperative Befunde (MRA-basierte Klassifikation nach Hefti et al. [19])

\begin{tabular}{l|l}
\hline Graduierung & MRA-Beschreibung \\
\hline Grad I & $\begin{array}{l}\text { Kleine Signaländerung ohne klare Begrenzung } \\
\text { Das osteochondrales Fragment mit klaren Rändern, jedoch ohne Flüssigkeit } \\
\text { zwischen Fragment und darunter liegendem Knochen }\end{array}$ \\
\hline Grad III & $\begin{array}{l}\text { Das Kontrastmittel ist teilweise zwischen Fragment und darunter liegenden } \\
\text { Knochen sichtbar }\end{array}$ \\
\hline Grad IV & $\begin{array}{l}\text { Das Kontrastmittel umgibt das Fragment vollständig. Das Fragment befindet } \\
\text { sich in situ und hat sich lokal nicht gelöst }\end{array}$ \\
\hline Grad V & Das Fragment ist vollständig gelöst und disloziert (freier Gelenkkörper)
\end{tabular}

die OD regelhaft nur an Teilabschnitten auf. Die avaskulären juvenilen Knochennekrosen sind meist selbstlimitierende Erkrankungen mit besserer Prognose verglichen mit der OD [50].

\section{Klinisches Erscheinungsbild}

Die meisten Patienten beschreiben einen schleichenden Beginn. Schmerzen werden häufig im lateralen Ellenbogen angegeben, können aber auch generalisiert das gesamte Gelenk betreffen. In der Regel werden belastungsabhängige Beschwerden beschrieben, welche durch adäquate Ruhigstellung regredient verlaufen können. Ein Extensionsdefizit ist häufig eines der Initialsymptome und sollte auch ohne einen radiologischen Nachweis freier Gelenkkörper als erstes Warnzeichen betrachtet werden. Aufgrund dieser unspezifischen Symptomatik werden Patienten häufig spät diagnostiziert. Sind bis dahin

\section{Radiologische Bildgebung und Klassifikationen}

Wie bei den allermeisten orthopädischen Krankheitsbildern stellt das konventionelle Röntgenbild auch bei der OD die Bildgebung der ersten Wahl dar. Im klinischen Alltag wird bei einem Patienten mit dem oben genannten unspezifischen Beschwerdebild primär eine klassische Ellenbogenaufnahme in 2 Ebenen veranlasst. Detektiert man dadurch zystische Läsionen z.B. am Capitulum im Sinne einer Transparenzsteigerung besteht der hochgradige Verdacht auf eine OD. Je höhergradiger die OD bereits vorliegt, können freie Gelenkkörper z. B. in der Fossa olecrani, ein abgeflachtes Capitulum, ein vergrößerter Radiuskopf oder auch Osteophyten sichtbar werden [49]. Zur besseren Darstellung des Capitulums wird zusätzlich die anteroposteriore Aufnahme in $45^{\circ}$ Flexion und $30^{\circ}$ Außenrotation empfohlen [31]. Gerade in früheren Stadien ist die Beurteilung des osteochondralen Defekts dadurch einfacher.

Bezüglich der konventionellen Röntgendiagnostik hat sich die Klassifikation nach Minami [50] durchgesetzt. Diese teilt die OD bezogen auf ihr Röntgenbild in drei Grade ein (• Tab. 1).

Als Diagnostikum zur Evaluation der Fragmentstabilität hat die Sonographie an Aufmerksamkeit ebenfalls gewonnen. Die Unterscheidung zwischen instabilen und stabilen Fragmenten zeigt sich hierbei durch eine chondrale Konturunterbrechung, wie bereits in mehreren Studien belegt werden konnte $[31,57]$.

Goldstandard bleibt die Magnetresonanztomographie (MRT). Für die Beurteilung der Größe, der exakten Lokalisation, aber auch der Stabilität hat sich die MRT als sensitivstes Verfahren etabliert. Die Klassifikation nach Dipaola et al. [16] teilt die OD in 4 Grade ein, wobei Grad II noch in a und b differenziert ist. Hierbei liegt eine Instabilität $\mathrm{ab}$ Grad IIb vor, bei der sich bildmorphologisch eine Separierung eines Teilfragments durch ein hyperintensens Signal interfragmentär oder zwischen Fragment und subchondralem Knochen in der T2Wichtung darstellt (• Abb. 1; • Tab. 2).

Hefti et al. [19] publizierten eine MRTbasierte Klassifikation und teilten die os- 
teochondrale Läsion dabei in 5 Stadien ein (•Tab. 3). Auch in dieser Einteilung existiert der Sprung vom instabilen und stabilen Fragment zwischen Stadium eins und zwei und ist ebenfalls über Kontrastmittel zwischen Knorpel und subchondralen Knochen definiert. Prinzipiell gilt ein Knorpeldefekt als instabil, wenn er sich von dem subchondralen Knochen gelöst hat. Die Frage nach der Stabilität des Fragments hat große therapeutische Signifikanz, da hier der Cut-off zwischen konservativer und operativer Therapie definiert ist.

Bei der Auswertung von MRT-Bildern des Ellenbogens sollte man den sog. Pseudodefekt beachten, um falsch-positive Diagnosen zu vermeiden. Der Pseudodefekt beschreibt anatomische Gegebenheiten des distalen Humerus, die in der Bildgebung fälschlicherweise als Defekte, osteochondrale Läsionen oder Impressionsfrakturen imponieren können. Für die OD ist dies v.a. der erkennbare Übergang von posteroinferiorem, artikulärem und extraartikulärem Knorpel am lateralen Capitulum. Nebst dem Fehlen eines Knochenmarködems ist die OD überwiegend anteriorer gelegen als es der Pseudodefekt suggeriert [39].

Die direkte MR-Arthrographie, also intraartikuläre Kontrastmittel(KM)Injektion während einer MRT, konnte die Sensitivität der Untersuchung in Studien erhöhen [26]. Das intraartikuläre KM bewirkt eine Aufdehnung des Gelenks. Ebenso werden separierte, instabile Fragmente mit KM umspült und sind dadurch sicherer beurteilbar. Gegen dieses Verfahren sprechen die Invasivität und das erhöhte Infektionsrisiko aufgrund der intraartikulären Punktion.

Die Computertomographie (CT) dient insbesondere der operativen Planung bei größeren Defektsituationen. Nebst der operativen Planung unterstützt sie die Detektion von bislang im nativen Röntgenbild nicht visualisierten freien Gelenkkörpern. Die Erweiterung um eine CT-Arthrographie (CTA) erzielte in Untersuchungen ähnlich gute Ergebnisse wie die Arthrographietechnik via MRT [20, 55]. Da die Untersuchung kürzer ist, kommt es zudem seltener zu Bewegungsartefakten. Nebst der gleichen Invasivität ist v.a. bei den meist

Obere Extremität 2020 · 15:273-283 https://doi.org/10.1007/s11678-020-00604-x

( ) Der/die Autor(en) 2020

\section{T. Khakzad $\cdot$ K. Thiele}

\section{Osteochondrosis dissecans des Ellenbogengelenks}

\section{Zusammenfassung}

Die Osteochondrosis dissecans (OD) ist eine weiterhin nicht vollständig verstandene Erkrankung, die in verschiedenen Gelenken auftreten kann. Der Ellenbogen, vornehmlich das Capitulum, stellt den zweithäufigsten Manifestationsort der OD dar. Auch wenn die OD eine seltene Erkrankung ist, erzeugt sie bei den meist jungen Patienten einen hohen Leidensdruck. Schmerzen, Bewegungseinschränkungen bis hin zur vollständigen Destruktion des Ellenbogengelenks können resultieren. Die unspezifischen Symptome der OD sorgen für eine häufig verspätete Diagnosestellung. Allgemeingültige Therapieempfehlungen speziell für den Ellenbogen sind rar. Die geeignete Therapiewahl hängt dabei entscheidend von dem Schweregrad und der Qualität der osteochondralen Läsion ab. Ebenso ist die genaue Beschreibung und Klassifikation des Defekts aufgrund unterschiedlichster Klassifikationssysteme aktuell erschwert. Diese basieren meist auf bildgebenden Verfahren wie dem nativem Röntgenbild oder der Magnetresonanzto- mographie. Die konservative Therapie ist nur bei stabilen Läsionen empfohlen. Sie beinhaltet eine mehrmonatige Sportkarenz und manuelle Therapie bis zur Beschwerdelinderung. Das operative Spektrum ist groß und reicht von arthroskopischem Débridement und Mikrofrakturierung, offenen Umstellungsosteotomien, neueren autologen Verfahren wie der autologen Chondrozytentransplantation (ACT) und der matrixinduzierten Chondrogenese (AMIC) bis hin zum osteochondralen Transfer (OATS). Ist die Indikation gut gestellt, lassen sich für die meisten Therapien hohe Erfolgsquoten generieren. Mit diesem Übersichtsartikel wurde der Versuch unternommen, die jeweiligen Indikationsschwerpunkte aus der aktuellen Literatur zu extrahieren und eine übersichtliche Behandlungsempfehlung vorzulegen.

Schlüsselwörter

Mikrofrakturierung · OATS · Klassifikation . Algorithmus · Débridement

\section{Osteochondritis dissecans of the elbow joint}

\section{Abstract}

Osteochondritis dissecans (OD), which can occur in various joints, is a disease that is not fully understood. The elbow, more precisely the capitellum, is the second most common place of OD manifestation. Although $\mathrm{OD}$ is a rare disease, it is associated with a high level of suffering in the mostly young patients. Pain, restriction of movement up to complete destruction of the elbow joint can result. The unspecific symptoms of OD mean that diagnosis is often delayed. General therapy recommendations, especially for the elbow, are rare. The appropriate choice of therapy depends crucially on the severity and quality of the osteochondral lesion. The exact description and classification of the defect is currently difficult due to the numerous classification systems. These are mostly based on imaging methods such as native $x$-rays or magnetic resonance imaging (MRI). Conservative therapy, which is only recommended for stable lesions, includes several months of no sports and manual therapy to alleviate symptoms. The surgical spectrum is large and ranges from arthroscopic debridement and microfracture, open conversion osteotomies, newer procedures such as autologous cartilage transplantation (ACT) and autologous matrix-induced chondrogenesis (AMIC) to osteochondral autologous transplantation system (OATS). If the indication is well established, high success rates can be obtained for most therapies. With this review article, the respective main indications were extracted from the current literature and a treatment recommendation is presented.

Keywords Microfracturing · OATS · Classification . Algorithm · Débridement 

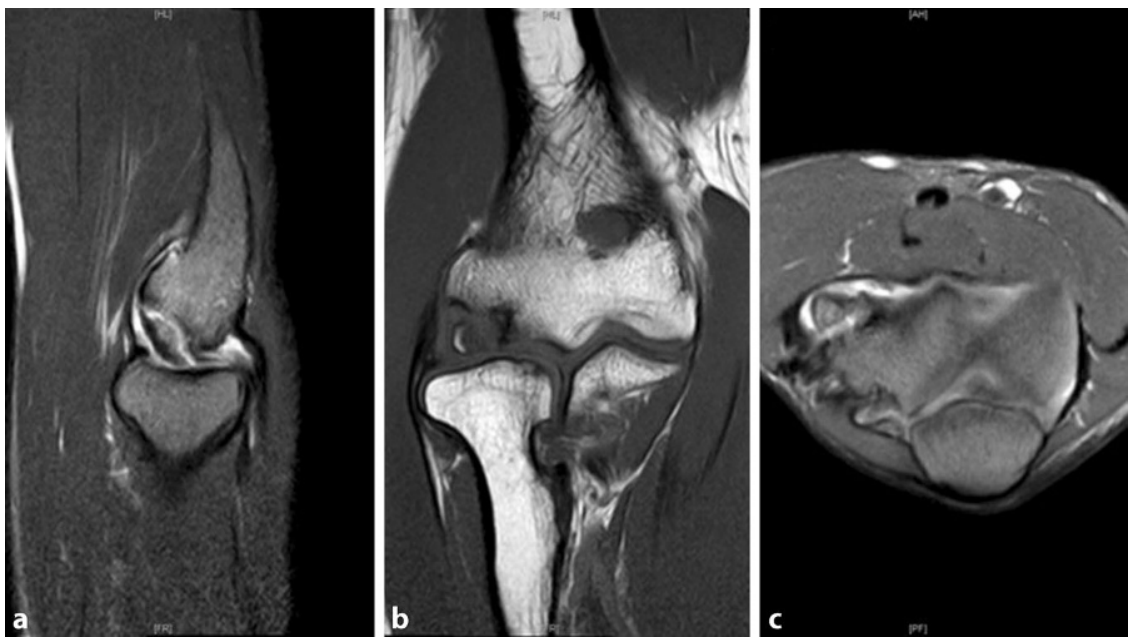

Abb. 1 ॥ Darstellung eines rechten Ellenbogengelenks im MRT mit einem großen osteochondralen Fragment im Bereich des Capitulums: Defekt in koronarer, sagittaler und axialerEbene. Die technischen Aufnahmeparameter können bei Fremdimport des MRT nicht angegeben werden

jungen Patienten die Strahlenbelastung ein zu überdenkender Aspekt, der gegen die CT-basierenden Verfahren sprechen kann.

Es gibt zahlreiche Klassifikationen osteochondraler Defekte. Zur Beschreibung von Größe, Graduierung und Lokalisation von chondralen und osteochondralen Defekten dient, auch am Ellenbogen, die einst für das Kniegelenk entworfene Klassifikation der International Cartilage Repair Society (ICRS; - Tab. 4; [24]).

Baumgartner et al. [2] entwickelten eine arthroskopische Klassifikation. Sie teilen dabei die makroskopische Beschaffenheit des Knorpels in 5 Qualitäten ein. Aus diesen können therapeutisch-operative Schritte abgeleitet werden (• Tab.5).

Unabhängig von der ärztlichen Spezialisierung, der verwendeten Klassifikation oder der Berufserfahrung zeigt sich eine hohe Variabilität in der Interpretation der radiologischen Bildgebung, wie Bexkens et al. [8] in ihrer Forschungsarbeit herauskristallisieren konnten. Sie empfehlen die Nutzung einfacher Klassifikations- und Entscheidungskriterien (z.B. stabil vs. instabil) gerade im Hinblick auf eine invasive operative Therapie.

\section{Therapie}

Die geeignete Behandlung osteochondraler Läsionen wird aufgrund der spärlichen Primärliteratur weiterhin kontrovers diskutiert. Wissenschaftlicher Konsens ist ein stadiengerechtes Vorgehen. Während stabile Läsionen konservativ behandelt werden sollten, ist das geeignete chirurgische Verfahren sowohl vom Stadium als auch anderen Indikationsschwerpunkten abhängig.

\section{Konservative Therapie}

Wissenschaftliche Arbeiten zur konservativen Behandlung der OD sind rar. Einigkeit besteht in der Tatsache, dass hohe Erfolgsraten nur bei stabilen Läsionen möglich sind [31, 32, 49]. Prinzipiell basiert die Therapie auf der Reduktion des Aktivitätsniveaus des betroffenen Ellenbogens und dem Vermeiden von repetitivem Stress, insbesondere Valgusstress sowie axiale Krafteinleitung. Dies beinhaltet eine strenge Sportkarenz für armbetonte Sportarten, wie sie häufig von den Betroffenen ausgeübt werden (Wurfsportarten, Gewichtheben etc.). Einige Autoren empfehlen eine kurze Phase der Ruhigstellung von bis zu 6 Wochen [5, 14]. Dies kann besonders in der Akutphase zur Schmerzreduktion sinnvoll sein und mit der medikamentösen Einnahme von Analgetika der WHO-Klasse I einhergehen. Dennoch eine evidenzbasierte Empfehlung zur Ruhigstellung fehlt [50]. Während einige Autoren eine vollständige Ruhigstellung empfehlen, postulieren andere Arbeiten Orthesen, die ein reduziertes Bewegungsausmaß zulassen, um eine konsekutive Steifigkeit zu vermeiden.

Nach der initialen Ruhigstellung sollte zur Prävention der Steifigkeit und bis zur Konsolidierung der Symptome eine Physiotherapie folgen. Auch wenn hier genaue Behandlungsvorschläge fehlen, ist das Vermeiden von mechanischem Stress auf das Ellenbogengelenk bei der physiotherapeutischen Beübung empfohlen. Entlastung durch passive Bewegungsübungen und manuelle Therapie mit Traktion sind aktuell die gängige Praxis.

\section{》) Die konservative Therapie kann bei stabilen Läsionen hohe Erfolgsraten erzielen}

Matsuura et al. [32] untersuchten das Outcome von 176 konservativ behandelten Patienten mit einer stabilen OD. Die konservative Therapie wurde als mindestens 6-monatige Vermeidung von schweren Lasten des betroffenen Ellenbogens definiert. Die Heilungsraten der nicht-complianten Patienten lag mit 22,3\% deutlich unter denen der complianten Patienten mit 84,2\%. Die Patienten mit einer Stadium I Läsion, also ohne sichtbare Fragmentierung mit reiner Transparenzerhöhung im Röntgenbild, konnten Heilungsraten von 90,5\% und die mit Stadium-II-Läsionen, also einer stabilen, nicht dislozierten Fragmentbildung, zeigten Heilungsraten von 52,9\%. Die mittlere Heilungszeit der StadiumI-Gruppe wurde mit 14,9 Monaten und der Stadium-II-Gruppe mit 12,3 Monaten angegeben. Als „Heilung“ wurde die Konsolidierung im nativen Röntgenbild bezeichnet.

Eine ebenfalls auf das native Röntgenbild verweisende Arbeit ist die von Mihara et al. [33]. Sie untersuchten die Heilungsraten von insgesamt 39 Baseballspielern. Auch hier wurde in die oben genannten Stadien eingeteilt und Patienten mit Stadium I und II, also einer stabilen Läsion, konservativ behandelt und evaluiert. Mihara et al. schließen neben demographischen Charakteristika die Reife der Wachstumsfuge in ihre Auswertung mit ein. Von den 17 Patienten mit offener Wachstumsfuge heilten 
Tab. 4 Klassifikationssysteme der Osteochondrosis dissecans für bildgebende Verfahren als auch intraoperative Befunde (Klassifikation nach ICRS [International Cartilage Repair Society]; [24])

\begin{tabular}{|l|l|l|}
\hline Graduierung & Status & Defektmorphologie \\
\hline Grad I & Normal & Keine \\
\hline Grad II & Fast Normal & $\begin{array}{l}\text { Oberfächliche Läsionen: Erweichungen }(\mathrm{A}) \text { und/oder ober- } \\
\text { flächliche Fissuren und Risse (B) }\end{array}$ \\
\hline Grad III & Abnormal & $\begin{array}{l}\text { Läsionen mit einer Tiefenausdehnung von <50\% der Knor- } \\
\text { peldicke }\end{array}$ \\
\hline Grad III & $\begin{array}{l}\text { Ausgeprägt Ab- } \\
\text { normal }\end{array}$ & $\begin{array}{l}\text { Knorpeldecke mit Tiefenausdehnung von }>50 \% \text { Knorpel- } \\
\text { dicke oder Ausdehnung in den subchondralen Knochen } \\
\text { sowie Blasenbildung }\end{array}$ \\
\hline Grad IV & $\begin{array}{l}\text { Ausgeprägt Ab- } \\
\text { normal }\end{array}$ & $\begin{array}{l}\text { Osteochondrale Läsion mit Ausdehnung bis in die subchon- } \\
\text { drale Knochenplatte bzW. in den trabekulären Knochen }\end{array}$ \\
\hline
\end{tabular}

Tab. 5 Klassifikationssysteme der Osteochondrosis dissecans für bildgebende Verfahren als

auch intraoperative Befunde (arthroskopische Einteilung nach Baumgarten et al. [2])

\begin{tabular}{l|l}
\hline Graduierung & Beschreibung \\
\hline Grad I & Knorpeloberfläche glatt, intakt, aber weich \\
\hline Grad II & Knorpeloberfläche aufgeraut \\
\hline Grad III & Knorpeloberfläche mit Fissuren \\
\hline Grad IV & Knorpel mit Fransen und oder freiem Knochen \\
\hline Grad V & Lose Knorpelteile/dislozierte Fragmente
\end{tabular}

16 letztendlich aus. Von den 22 Patienten mit geschlossener Wachstumsfuge konnte nur in $50 \%$ eine Heilung nachgewiesen werden. 30 der 39 Patienten hatten Stadium-I-Läsionen und 9 Stadium-II-Läsionen, von denen lediglich ein Patient (1/9) geheilt werden konnte. Bei genauer Betrachtung fällt auf, dass aus der Gruppe mit geschlossenen Wachstumsfugen, fast die Hälfte höhergradige Läsionen aufwies und daher das Ergebnis der Vergleichsgruppen etwas schmälert. Nichtsdestotrotz scheint die offene Wachstumsfuge als Prognosekriterium für die konservative Therapie berechtigt zu sein. Takahara et al. [48] bestätigen diesen Fund in ihrer retrospektiven Analyse von insgesamt 106 Patienten, von denen 36 konservativ durch Sportkarenz therapiert wurden. Neben der offenen Wachstumsfuge sind auch das Bewegungsausmaß zum Zeitpunkt der Diagnosestellung als auch die Compliance positive Prognosefaktoren für das Gelingen der konservativen Therapie.

Prognosefaktoren für die konservative Therapie sind Gegenstand aktueller Forschungsarbeiten wie der von Funakoshi et al. [17]. Unter Einbeziehung mehrerer radiologischer Bildgebungsverfahrung (konv. Röntgen, CT und MRT) gehen Funakoshi et al. auf die Vergröße- rung des Radiuskopfes sowie der Differenz des Skelettalters nach Dimeglio et al. [15], ein modifiziertes Scoringsystem nach Sauvagrain, zwischen geheilter und nicht-geheilter OD ein. Sauvagrain et al. publizierten ein Scoringsystem mit insgesamt 27 Punkten, aufgeteilt auf vier anatomische Strukturen des Ellenbogens [43]. In der Regressionsanalyse von Funakoshi et al. [17] konnte ein hoher skelettaler Alters- und Entwicklungsunterschied, insbesondere mit differenten fortgeschrittenen Wachstum des Radiuskopfes, des lateralen Kondylus als auch des Olekranons als negative Prädiktoren für die konservative Therapie extrahiert werden.

Die Arbeitsgruppe von Niu et al. [36] arbeitete ebenfalls weitere Entscheidungskriterien auf Grundlage einer Regressionsmodelanalyse aus. Dafür untersuchten sie retrospektiv insgesamt 89 Patienten (93 Ellenbogengelenke) mit einer OD des Capitulums nach. Nebst der MRT-basierten Hefti-Klassifikation und weiteren möglichen Einflussfaktoren wurden auch MRT-morphologische Charakteristika wie die Reife der Wachstumsfuge, zystische Veränderungen, die Defektlokalisation und die Relation zwischen Defektgröße bezogen auf die Gesamtgröße des Capitulums in das Regressionsmodel einbezogen [19]. Den Ergebnissen folgend hatten das Geschlecht, die Sportart, der dominante Arm sowie die initiale Beweglichkeit keinen Einfluss auf die Heilungschancen der konservativen Behandlung. Vielmehr scheinen eine kurze Symptomdauer vor Beginn der Therapie, das Fehlen zystischer Strukturen als auch die relative Defektgröße bezogen auf das individuell ausgemessene Capitulum prognosebestimmend zu sein. Die Reife der Wachstumsfuge beeinflusste die Heilungsprognose nicht signifikant, was verglichen mit den oben zitierten Studien überrascht. Die Gesamtheilungsrate wurde deutlich geringer mit $53 \%$ angeben bei einer mittleren Therapiezeit mit 8,3 Monaten [36].

Die Variabilität der zitierten Studien bezogen auf das Studiendesign, die Begrifflichkeit und Definition von Heilung und deren Bemessungsmethoden (konv. Röntgen, MRT, Symptomverlust) sowie die unterschiedlich verwendeten Klassifikationssysteme lassen einen direkten Vergleich nicht zu. Zusammenfassend lässt sich dennoch definieren, dass eine früh erkannte stabile Läsion häufig von der konservativen Therapie profitieren kann. Bei dem Vorhandensein skelettaler Reifeunterschiede, v. a. eines vergrößerten Radiuskopfes, dem Auftreten von zystischen Veränderungen und langer prätherapeutischer Symptomdauer sollte selbst bei stabilen großen Läsionen ein konservatives Prozedere hinterfragt werden.

\section{Operative Therapie}

Aufgrund der geringen Regenerationsfähigkeit hyalinen Knorpels stellt die operative Behandlung von osteochondralen Läsionen eine große Herausforderung dar. Innerhalb der letzten Dekaden wurden mehrere operative Verfahren entwickelt. Die Entscheidung, welches Verfahren empfohlen werden sollte, ist von der Defektqualität abhängig.

\section{Débridement und Mikrofrakturierung}

Beim Débridement werden freie Knorpellamellen entfernt und der Knorpel getrimmt. Innerhalb des arthroskopi- 


\section{Übersicht}
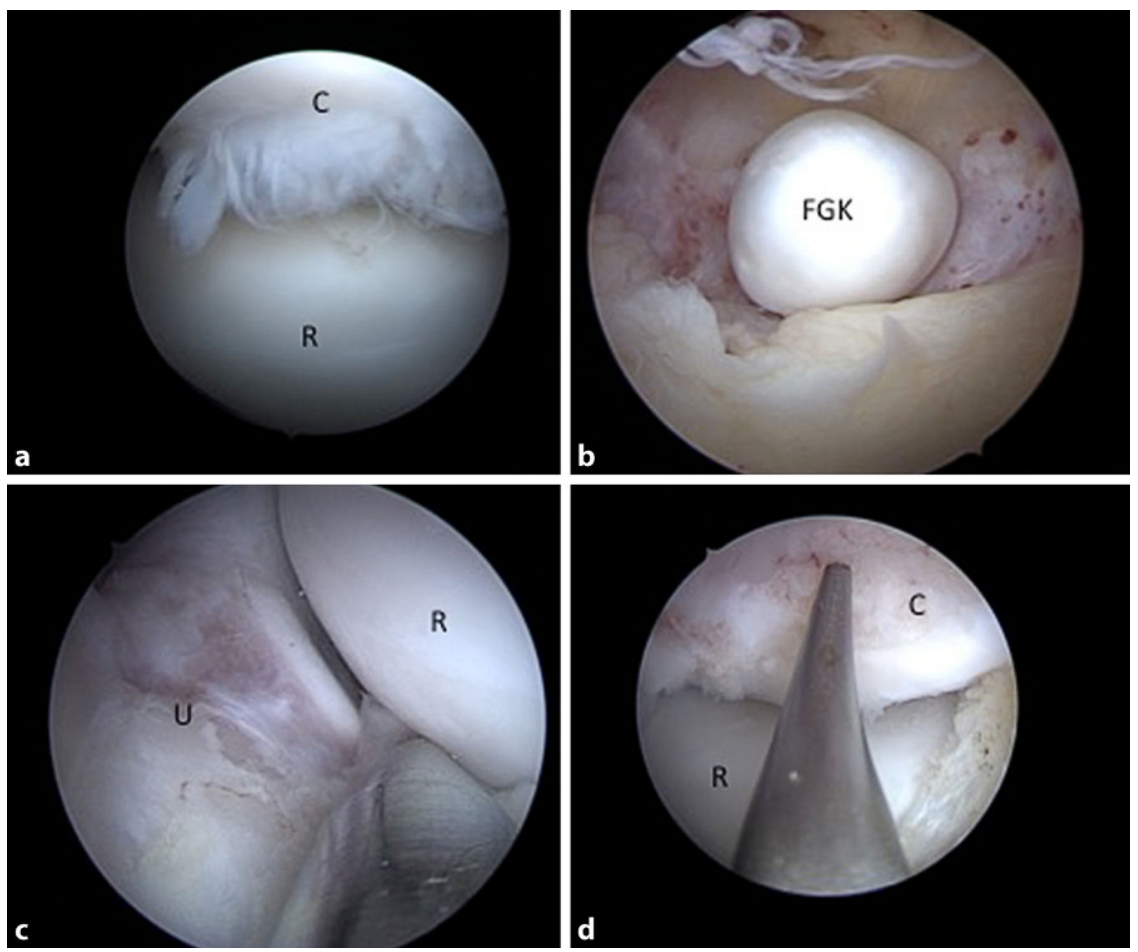

Abb. $2 \Delta$ Arthroskopische Darstellung eines osteochondralen Defekts sowie dem Therapieverfahren der Mikrofrakturierung mit Ansicht aus dem posterolateralen Portal mittels $30^{\circ}$-Optik: a Darstellung des chondralen Defekts im Bereich des Capitulums, b Darstellung eines freien Gelenkkörpers, cradioulnare Stabilitätsprüfung, d Mikrofrakturierung des chondralen Defekts.CCapitulum, RRadiusköpfchen, U Ulna [„,bare area“], FGK freier Gelenkkörper)

schen Eingriffs kann das Débridement mit der Entfernung freier Gelenkkörpern und einer Synovektomie erfolgen. Mehrere Studien belegen den kurzfristigen Erfolg des Eingriffs $[11,12,40]$. Dennoch hat das Débridement keinen Einfluss auf die Neubildung hyalinen Knorpels, so dass ein Fortschreiten der radiohumeralen Arthrose nicht aufgehalten wird. Ueda et al. [52] untersuchten retrospektiv die Progression der radiohumeralen Arthrose an 38 Patienten nach Débridement und Fragmentresektion. Bei einer Defektgröße von $>50 \%$ der Gelenkfläche zeigte sich bei dem Patientenkollektiv eine signifikante Progression der Osteoarthrose um einen Grad in einem Beobachtungszeitraum von 5-12 Jahren.

Die Mikrofrakturierung ergänzt das Débridement zusätzlich als knochenmarkstimulierendes Verfahren (• Abb. 2) Das Einschlagen von Stößeln in den Subchondralraum hat sich im Vergleich zu anderen knochenmarkstimulierenden Verfahren wie dem Aufbohren oder der Abrasionsarthroplastik durchgesetzt. Allen gemein ist das Schaffen eines Blutkoagels aus den eröffneten Subchondralgefäßen, der sich mit der Zeit zu einem Fibrinkoagel und letztendlich zu Faserknorpel transformiert. Die Einhaltung eines restriktiven Nachbehandlungsschemas ist für den Erfolg obligat, um den fragilen Fibrinclot vor Druck- und Scherkräften zu schützen.

Die Mikrofrakturierung wurde erstmals durch Steadman et al. [46] beschrieben. Der neu entstehende Faserknorpel ist im Vergleich zum hyalinen Knorpel von minderer Belast- und Haltbarkeit. Kniegelenkstudien zeigten, dass bereits nach Monaten eine Degeneration des neuentstandenen Faserknorpels nachweisbar ist, sodass nach eingangs hervorragenden Kurzzeitergebnissen im Verlauf auch eine klinische Verschlimmerung der Symptome folgte [27]. Steadman et al. [46] beschrieben die Technik der Mikrofrakturierung für rein chondrale Defekte, was auch die Limitierung der Therapieempfehlung aufzeigt. Tiefe osteochondrale Defekte können durch eine chondral adressierte Therapie nicht adäquat behandelt werden, da der defekte subchondrale Knochen nicht wiederhergestellt wird. Bojanic et al. [9] plädieren in ihrer Fallserie für eine Grenztiefe von $3 \mathrm{~mm}$ subchondral des Defekts. Gleichsam wird die Mikrofrakturierung nur bei Defektgrößen $<50 \%$ der kapitulären Gelenkfläche, die „contained“, also mit vitalem Knorpel umrandet sind, empfohlen. Ist der Defekt größer oder überschreitet er die laterale Begrenzung, zeigt sich ein schlechteres postoperatives Ergebnis.

Dennoch, sind die Indikationskriterien erfüllt, kommt es in bis zu $80 \%$ der Fälle zu einer suffizienten fibrokartilaginären Neubildung und bei mittelfristigen Verlaufszeiten zu teils sehr guten Ergebnissen. Auch wenn nur ca. zwei Drittel der Patienten ihr vorbestehendes sportliches Niveau wieder erreichen, so schaffen es nach reiner Mikrofrakturierung fast $90 \%$ wieder zurück in eine sportliche Aktivität nach ca. 5 Monaten $[6,9,12]$.

\section{Fragmentrefixation}

Die Fragmentrefixation kann offen oder arthroskopisch angegangen werden (- Abb. 3). Meist wird das Fragment vollständig gelöst, der Grund kürettiert und dann unter Zuhilfenahme chirurgischer Produkte refixiert. Für die Refixation eignen sich Herbert-Schrauben, KirschnerDrähte, bioabsorbierbare Pins ebenso wie Knochenspanbolzen oder dynamische Klammersysteme. Empfohlen wird die Refixation bei instabilen Läsionen Grad II nach Dipaola et al. [16] oder Grad II-III nach ICRS-Klassifikation. Eine weitere Bedingung zur Refixation stellt eine intakte laterale Wandbegrenzung dar. Sind die Indikationskriterien erfüllt, lassen sich gute Ergebnisse realisieren wie mehrere Fallstudien suggerieren. In einem systematischen Review zeigten sich nach einem halben Jahr 75-100\% der refixierten Fragmente konsolidiert. Ohne einen Niveauunterschied zu messen, konnten den Ergebnissen folgend, bis $\mathrm{zu} 91,4 \%$ wieder sportlich aktiv werden [28].

\section{ACT und AMIC}

Die Transplantation von Chondrozyten stellt bei chondralen Defekten aktuell am Knie und Sprunggelenk eine wirkliche Alternative dar. Bei diesem Verfahren 

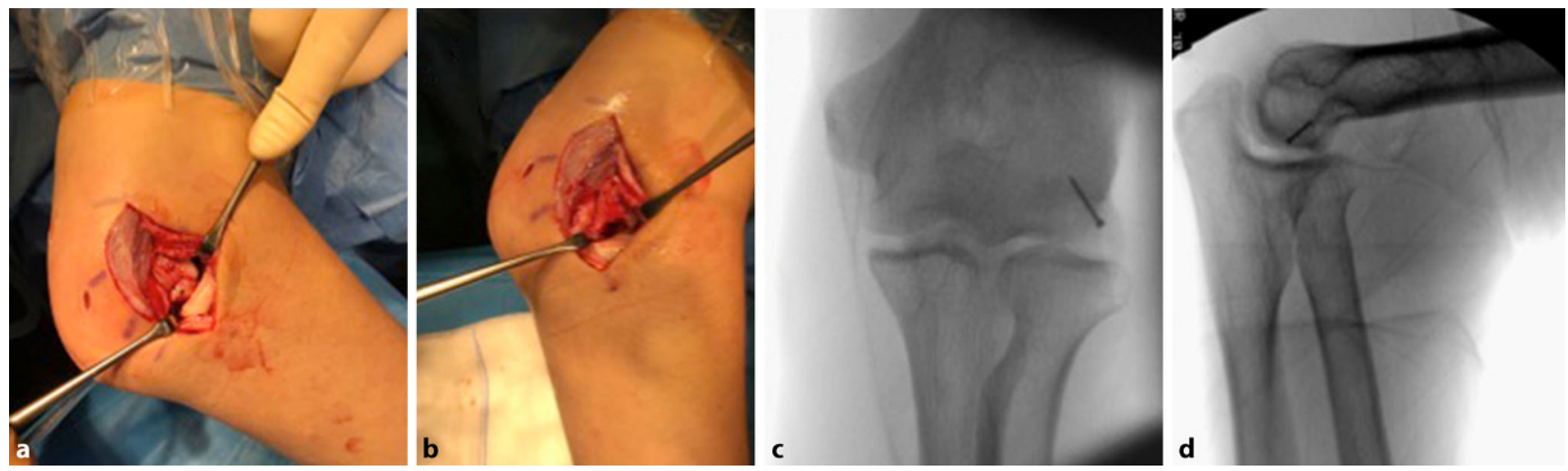

Abb. 3 A Darstellung einer Fragmentrefixation mittels HCS-Schraube und ACT-Implantation (autologe Chondrozytentransplantation, zweizeitiges Vorgehen) bei einem Grenzbefund. Aufgrund des jungen Alters des Patienten wurde diese Therapie bevorzugt eingesetzt: a Intraoperatives Bild des Defekts nach Zugang über das Kocher-Intervall, b Darstellung der Defektsituation nach Entnahme des Fragments, c Intraoperatives a.-p.-BV-Bild nach Refixation der osteochondralen Läsion mittels 1,5 HCS-Schraube, $\mathbf{d}$ Intraoperatives laterales BV-Bild nach Schraubenrefixation des Fragments
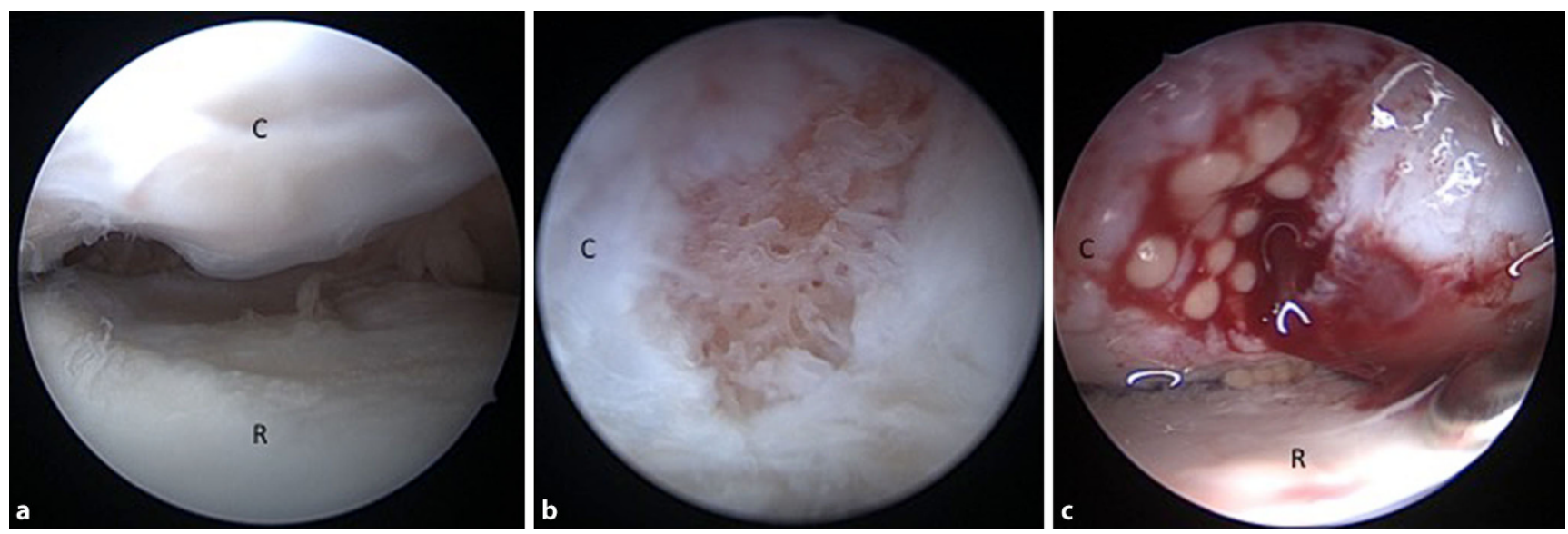

Abb. 4 ム Darstellung einer arthroskopischen ACT-Implantation (autologe Chondrozytentransplantation) 10 Wochen nach offener Fragmentrefixation (s. • Abb. 3) mit intraartikulärer arthroskopischer Darstellung des capitulären Defekts bei maximaler Flexion (Ansicht über das posterolaterale Portal mit 30-Optik): a Intraoperative Darstellung des Capitulums von dorsal im Zustand nach Fragmentrefixation, b intraoperative Darstellung im Rahmen der Rearthroskopie mit Sicht auf den débridierten chondralen Defekt des Capitulums, c intraoperative Darstellung im Rahmen der Rearthroskopie nach ACT in die chondrale Defektzone des Capitulums. Das Kontroll-MRT steht zum jetzigen Zeitpunkt noch aus. C Capitulum, $R$ Radiusköpfchen)

werden Chondrozyten meist aus der Femurkondyle außerhalb der Belastungszone explantiert, im Labor gezüchtet und nach einigen Wochen replantiert. Die autologe Chondrozytentransplantation (ATC; - Abb.4) wurde erstmals im Kaninchenmodel von Peterson et al. [38] erfolgreich publiziert. Dieses Verfahren adressiert nur den reinen Knorpelschaden. Besteht eine Läsion des subchondralen Knochens, so muss dieser zumindest durch, z.B. eine Spongiosaplastik, aufgefüllt werden. Die Arbeitsgemeinschaft (AG) „Geweberegeneration und Gewebeersatz" der Deutschen Gesellschaft für Orthopädie und orthopädischer Chirurgie (DGOOC) und der Deutschen
Gesellschaft für Unfallchirurgie (DGU) empfiehlt aktuell die ACT erst bei einem reinen chondralen Defekt und einer Defektgröße ab 3-4 $\mathrm{cm}^{2}$ für das Kniegelenk. Bei jungen, sportlich aktiven Menschen bereits ab einer Defektgröße von $2,5 \mathrm{~cm}^{2}$. Gleichsam ist sie nicht für einen flächenhaften Knorpelschaden im Sinne einer degenerativen Abnutzung, sondern für einen ICRS-III- bis -IVKnorpelschaden empfohlen, die meist traumatischer Genese sind [35]. Allein die bisher geforderte Defektgröße bestätigt den geringen Stellenwert der ACT in der Ellenbogenchirurgie aktuell. Auch wenn die ACT in der Kniechirurgie in die Empfehlungen Einzug genommen hat und sich vergleichsweise viele Studien dazu finden lassen, die deren Erfolg aufzeigen, fehlen genau diese für die Ellenbogenchirurgie.

Sato et al. [42] implantierten einem Patienten Chondrozyten im M-ACT-Verfahren, dem sog. matrixgekoppelten Verfahren, bei dem eine bioresorbierbare Substanz als Trägermaterial der Chondrozyten fungiert. Der 14-jährige Patient hatte in der Kontrollarthroskopie nach 24 Monaten kongruente Gelenkflächen. Er konnte sein anfängliches Extensionsdefizit von $15^{\circ}$ auf $5^{\circ}$ senken und war schmerzfrei. Patzer et al. [37] beschrieben die ACT am Ellbogen für weitere 3 Patienten. Ebenso stellt er die Möglichkeit der 


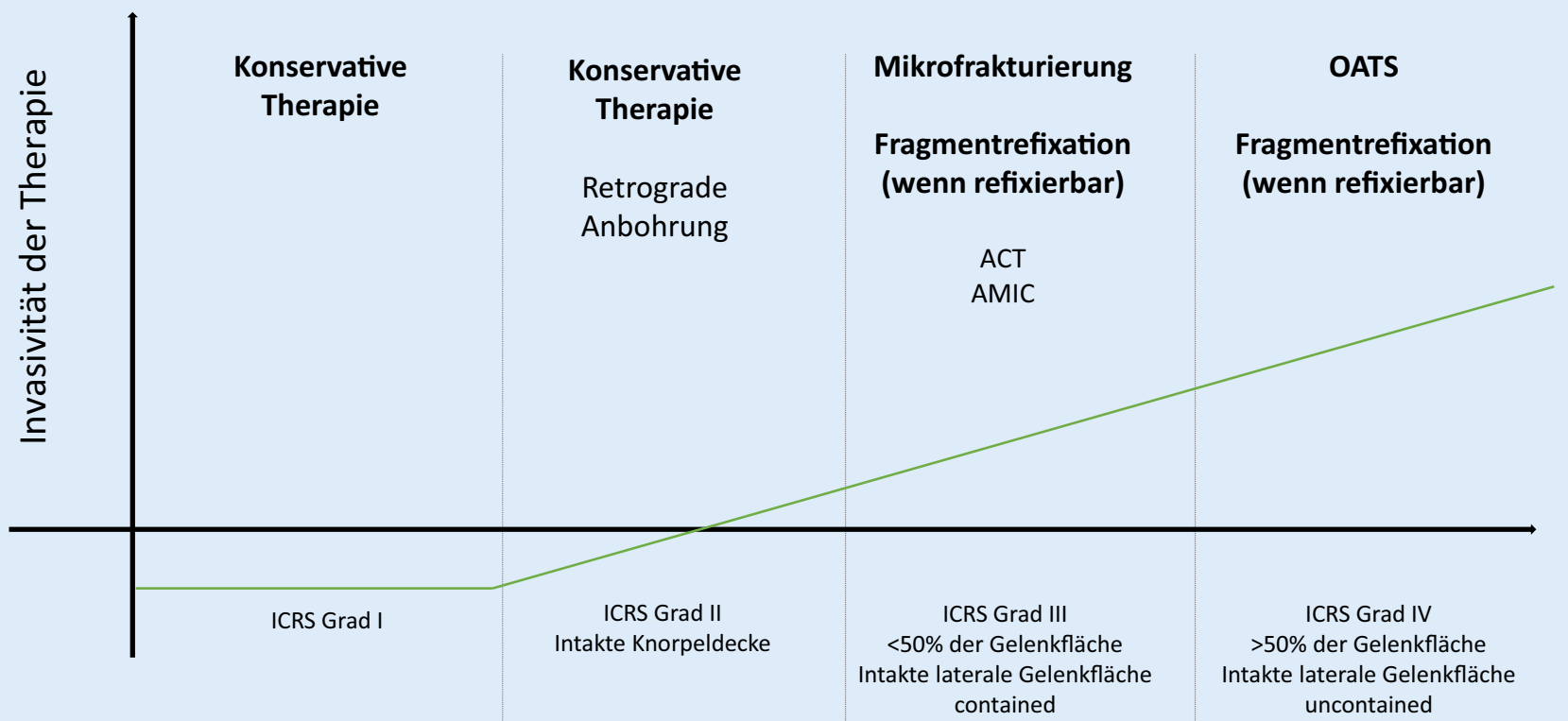

Schweregrad der Osteochondrosis dissecans

Abb. $5 \Delta$ Therapieempfehlung anerkannter Verfahren in Relation zum Schweregrad der osteochondralen Läsion

Chondrozytenentnahme aus der Olekranonspitze dar. Dieses würde einen zweiten operativen Zugangsweg zum Knie oder anderen Körperstellen obsolet machen.

Caldwell et al. [13] publizierten 2017 die arthroskopische Implantation von mikronisierten „Scaffolds“. Scaffold lässt sich als resorbierbares Biogerüst übersetzen, das die Knorpelzellen beherbergt und nach dem Auftragen eine hyaline Knorpelzellformation induziert. Durch die Errungenschaften des „tissue engineering " gibt es immer wieder innovative Therapieansätze. Die AMIC (autologe matrixinduzierte Chondrogenese) stellt auch für die Ellenbogenchirurgie in diesem Zusammenhang einen neuen, wenig erforschten Ansatz dar. Nach durchgeführter Mikrofrakturierung wird die Defektzone von einer azellulären Kollagenschicht bedeckt. Die einströmenden Stammzellen verweilen, eingeschlossen in die Kollagenmatrix in der Defektzone und fördern so die Integration und Transformation der Zellen zu Chondrozyten [3]. Auch hier bedarf es einer intakten subchondralen Situation.

\section{"Osteochondral autologous transplantation system" (OATS)}

Da ein Chondrozytentransfer nur rein chondrale Defekte wiederherstellen kann, ist die Transplantation osteochondraler Autografts die einzige rekonstruktive Therapie bei höhergradigen osteochondralen Defekten. Durch körpereigene Knorpel-Knochen-Zylinder wird der subchondrale Knochen rekonstruiert und mit einer hyalinen Knorpeldecke besetzt. Sie ist daher für hochgradige, instabile Läsionen (Grad III-IV nach Dipaola) und einer Tiefe bis zu $15 \mathrm{~mm}$ empfohlen [25]. Die OATSTherapie hat den Nachteil, dass diese Knorpel-Knochen-Zylinder aus einem Zweitgelenk entnommen werden müssen. Deren Verfügbarkeit stellt daher eine Limitation dar. Während bei der klassischen OATS die Zylinder bis $\mathrm{zu}$ $15 \mathrm{~mm}$ groß sind, werden bei einer Mosaikplastik, zu Ungunsten der Primärstabilität des osteochondralen Konstrukts deutlich kleinere Autografts transferiert. Die kleinen Knorpel-KnochenZylinder der Mosaikplastik erlauben es, die Gelenkkongruenz besser darzustellen. Es zeigte sich aber, dass der Anteil an Faserknorpel, der zwischen den Autografts entsteht, deutlich höher liegt als bei dem Transfer von einem $15 \mathrm{~mm}$ großen Autograft [29]. Gleichsam ist der operative Transfer deutlich erschwert. Forschungsarbeiten zeigten, dass ein Niveauunterschied der Zylinder von $0,5 \mathrm{~mm}$ ausreicht, um dort den Spitzendruck um $50 \%$ bei Belastung zu steigern. Die „herausragenden“ Zylinder sind gleichsam Scherkräften ausgesetzt, die das Gesamtergebnis gefährden und die Graftinkorporation erschweren. Es hat sich daher vermehrt die „klassische" OATS als Therapie durchgesetzt. Da bei dem Transfer größerer Autografts weniger Autograft-Zwischenraum (Interface) entsteht, ist der Anteil an gebildetem Faserknorpel geringer. Die Zylinder werden in Press-fit-Technik transferiert. Dies gestaltet die Operationstechnik deutlich einfacher verglichen mit der Mosaikplastik. Bei der Implantation muss genau auf die geometrischen Verhältnisse der Gelenkoberfläche geachtet werden. Bei kleinen Gelenkflächen wie dem Capitulum kann das herausfordernd sein. Schafft der Operateur es nicht, kongruente Gelenkflächen zu erzeugen, können degenerative Prozesse beschleunigt werden. Ist es hingegen gelungen, so zeigen mehrere Forschungsarbeiten gute bis exzellente Ergebnisse 
auf. $94 \%$ der Operierten schaffen es auf ein identisches Sportniveau zurückzukehren. Eine Rückkehr zur sportlichen Aktivität gelingt laut einer Metaanalyse von 24 Publikationen am besten nach der OATS-Prozedur [22, 30, 51, 54, 56]. In mehr als drei Viertel der Fälle (77\%) kann durch die OATS eine Restauration der Knorpelintegrität geschaffen werden. Die Graftinkorporationszeit beträgt ca. 4 Monate $[41,45]$. Kritisch wird weiterhin die Entnahmestelle diskutiert. In der Regel erfolgt die Entnahme aus der Trochlea am Kniegelenk (laterale und mediale Facette). Hier wird durch anatomische MRT-Studien eine Inkongruenz zwischen der artikulären Knorpelkurvatur von $1 \mathrm{~mm}$ zwischen Knie und Ellbogen beschrieben.

\section{》) Große osteochondrale De- fekte können die Transplantation osteochondraler Autografts erfordern}

Bei Läsionen $>15 \mathrm{~mm}$ wurde die Entnahme der kostoosteochondralen Verbindung der 5 . und 6 . Rippe als alternative Entnahmestelle aufgezeigt. Sato et al. [41] beschrieben auch für die Methode exzellente Ergebnisse. Bexkens et al. [7] kommen zu den Ergebnis, dass die Entnahme aus dem kostoosteochondralen Übergang weniger Hebedefektmorbidität erzeugt (1,6\%) im Vergleich zur Entnahme aus dem Kniegelenk (7,8\%).

\section{Behandlungsempfehlung}

Aufgrund der seltenen Inzidenz und den teils unterschiedlichen Studiendesigns fällt es schwer, einen evidenzbasierten Therapiealgorithmus zu deklarieren. Wir haben, mit unserem Therapieschema, den Versuch gemacht, anerkannte wissenschaftliche Daten in eine übersichtliche Behandlungsempfehlung darzustellen (• Abb. 5).

\section{Zusammenfassung}

Die OD des Ellenbogengelenks stellt ein seltenes Krankheitsbild dar, deren Ätiologie nicht vollends verstanden ist. Ein möglicher Therapiealgorithmus wird bestimmt durch die Fragmentstabilität, die osteochondralen Größenausdehnung sowie dem Containment des Defekts. Verschiedene Klassifikationssysteme basierend auf Röntgen, MRT und intraoperativen Befunden versuchen mittels Graduierung des Defekts eine gezielte Behandlung zuzuordnen.

\section{Fazit für die Praxis}

- Die konservative Therapie ist stabilen osteochondralen Läsionen vorbehalten.

- Prognostisch ungünstig für einen Heilungserfolg scheinen sich hierbei eine geschlossene Wachstumsfuge, zystische Strukturformationen, akzelerierter Knochenwachstum sowie die Größe des Defekts auszuwirken.

- Bei Versagen der konservativen Therapie besteht die Wahl zwischen ossär induktiven (Mikrofrakturierung, Débridement) und rekonstruktiven Operationsverfahren (OATS [„osteochondral autograft transfer system“], Fragmentrefixation).

- Neuere Therapieformen zur Regeneration von chondralen Defekten wie die Implantation von autologen Chondrozyten werden in einzelnen Fallbeschreibungen vorgestellt, jedoch stehen Langzeitergebnisse noch aus.

\section{Korrespondenzadresse}

\section{Dr. Kathi Thiele}

Centrum für Muskuloskeletale Chirurgie, Universitätsmedizin Berlin, Campus Virchow Augustenburger Platz 1, 13353 Berlin, Deutschland

Kathi.Thiele@charite.de

Funding. Open Access funding enabled and organized by Projekt DEAL.

\section{Einhaltung ethischer Richtlinien}

Interessenkonflikt. T. Khakzad und K. Thiele geben an, dass kein Interessenkonflikt besteht.

Für diesen Beitrag wurden von den Autoren keine Studien an Menschen oder Tieren durchgeführt. Für die aufgeführten Studien gelten die jeweils dort angegebenen ethischen Richtlinien.
Open Access. Dieser Artikel wird unter der Creative Commons Namensnennung 4.0 International Lizenz veröffentlicht, welche die Nutzung, Vervielfältigung, Bearbeitung, Verbreitung und Wiedergabe in jeglichem Medium und Format erlaubt, sofern Sie den/die ursprünglichen Autor(en) und die Quelle ordnungsgemäß nennen, einen Link zur Creative Commons Lizenz beifügen und angeben, ob Änderungen vorgenommen wurden.

Die in diesem Artikel enthaltenen Bilder und sonstiges Drittmaterial unterliegen ebenfalls der genannten Creative Commons Lizenz, sofern sich aus der Abbildungslegende nichts anderes ergibt. Sofern das betreffende Material nicht unter der genannten Creative Commons Lizenz steht und die betreffende Handlung nicht nach gesetzlichen Vorschriften erlaubt ist, ist für die oben aufgeführten Weiterverwendungen des Materials die Einwilligung des jeweiligen Rechteinhabers einzuholen.

Weitere Details zur Lizenz entnehmen Sie bitte der Lizenzinformation auf http://creativecommons.org/ licenses/by/4.0/deed.de.

\section{Literatur}

1. Barnes DA, Tullos HS (1978) An analysis of 100 symptomatic baseball players. Am J Sports Med 6:62-67. https://doi.org/10.1177/ 036354657800600205

2. Baumgarten TE, Andrews JR, Satterwhite YE (1998) The arthroscopic classification and treatment of osteochondritis dissecans of the capitellum. Am J Sports Med 26:520-523. https://doi.org/10.1177/ 03635465980260040801

3. Behrens P (2005) Matrixgekoppelte Mikrofrakturierung. Arthroskopie 18:193-197. https://doi. org/10.1007/s00142-005-0316-0

4. Bennett GE (1947) Shoulder and elbow lesions distinctive of baseball players. Ann Surg 126:107-110. https://doi.org/10.1097/00000658-19470700000008

5. van Bergen $\mathrm{CJ}$, van den Ende KI, Ten Brinke B, Eygendaal D (2016) Osteochondritis dissecans of the capitellum in adolescents. World J Orthop 7:102-108. https://doi.org/10.5312/wjo.v7.i2.102

6. Bexkens R, van den Ende KIM, Ogink PT et al (2017) Clinical outcome after arthroscopic debridement and microfracture for osteochondritis dissecans of the capitellum. Am J Sports Med 45:2312-2318. https://doi.org/10.1177/0363546517704842

7. Bexkens R, Ogink PT, Doornberg JN et al (2017) Donor-site morbidity after osteochondral autologous transplantation for osteochondritis dissecans of the capitellum: a systematic review and meta-analysis. Knee Surg Sports Traumatol Arthrosc 25:2237-2246. https://doi.org/10.1007/ s00167-017-4516-8

8. Bexkens R, Claessen FMAP, Kodde IF et al (2018) Interobserver reliability of radiographic assessment after radial head arthroplasty. Shoulder Elbow 10:121-127. https://doi.org/10.1177/ 1758573217719088

9. Bojanić I, Smoljanović T, Dokuzović S (2012) Osteochondritis dissecans of the elbow: Excellent results in teenage athletes treated by arthroscopic debridement and microfracture. Croat Med J 53:40-47. https://doi.org/10.3325/cmj.2012.53. 40

10. Bradley JP, Petrie RS (2001) Osteochondritis dissecans of the humeral capitellum. Diagnosis 
and treatment. Clin Sports Med 20:565-590. https://doi.org/10.1016/s0278-5919(05)70270-2

11. Brownlow HC, O'Connor-Read LM, Perko M (2006) Arthroscopic treatment of osteochondritis dissecans of the capitellum. Knee Surg Sports Traumatol Arthrosc 14:198-202. https://doi.org/ 10.1007/s00167-005-0623-z

12. Byrd JWT, Jones KS (2002) Arthroscopic surgery for isolated capitellar osteochondritis dissecans in adolescent baseball players: Minimum three-year follow-up. Am J Sports Med 30:474-478. https:// doi.org/10.1177/03635465020300040401

13. Caldwell PE, Auerbach B, Pearson SE (2017) Arthroscopic treatment of capitellum osteochondritis dissecans with micronized allogeneic cartilage scaffold. Arthrosc Tech 6:e815-e820. https://doi.org/10.1016/j.eats.2017.04.007

14. Churchill RW, Munoz J, Ahmad CS (2016) Osteochondritis dissecans of the elbow. Curr Rev Musculoskelet Med 9:232-239. https://doi.org/10. 1007/s12178-016-9342-y

15. Diméglio A, Charles YP, Daures J-P et al (2005) Accuracy of the Sauvegrain methodin determining skeletal age during puberty. J Bone Joint Surg Am 87:1689-1696. https://doi.org/10.2106/JBJS. D. 02418

16. Dipaola JD, Nelson DW, Colville MR (1991) Characterizing osteochondral lesions by magnetic resonance imaging. Arthrosc J Arthrosc Relat Surg 7:101-104. https://doi.org/10.1016/07498063(91)90087-e

17. Funakoshi T, Furushima K, Miyamoto A et al (2019) Predictors of unsuccessful nonoperative management of capitellar osteochondritis dissecans. Am J Sports Med 47:2691-2698. https://doi.org/10. $1177 / 0363546519863349$

18. Haraldsson S (1959) On osteochondrosis deformas juvenilis capituli humeri including investigation of intra-osseous vasculature in distal humerus. Acta Orthop Scand Suppl 38:1-232

19. Hefti F, Beguiristain J, Krauspe R et al (1999) Osteochondritis dissecans: a multicenter study of the European Pediatric Orthopedic Society. JPediatr Orthop B 8:231-245

20. Holland P, Davies AM, Cassar-Pullicino VN (1994) Computed tomographic arthrography in the assessment of osteochondritis dissecans of the elbow. Clin Radiol 49:231-235. https://doi.org/10. 1016/S0009-9260(05)81846-X

21. Hsu SH, Moen TC, Levine WN, Ahmad CS (2012) Physical examination of the athlete's elbow. Am J Sports Med 40:699-708. https://doi.org/10.1177/ 0363546511428869

22. Iwasaki N, KatoH, Ishikawa Jetal (2009) Autologous osteochondral mosaicplasty for osteochondritis dissecans of the elbow in teenage athletes. J Bone Joint Surg Am 91:2359-2366. https://doi.org/10. 2106/JBJS.H.01266

23. Kida Y, Morihara T, Kotoura Yet al (2014) Prevalence and clinical characteristics of osteochondritis dissecans of the humeral capitellumamongadolescent baseball players. Am J Sports Med 42:1963-1971. https://doi.org/10.1177/0363546514536843

24. Kohyama S, Ogawa T, Mamizuka N et al (2018) A magnetic resonance imaging-based staging system for osteochondritis dissecans of the elbow: a validation study against the international cartilage repair society classification. Orthop J Sports Med. https://doi.org/10.1177/2325967118794620

25. Kosaka M, Nakase J, Takahashi R et al (2013) Outcomes and failure factors in surgical treatment for osteochondritis dissecans of the capitellum. J Pediatr Orthop 33:719-724. https://doi.org/10. 1097/BPO.0b013e3182924662
26. Kramer J, Stiglbauer R, Engel A et al (1992) MR contrast arthrography (MRA) in osteochondrosis dissecans. J Comput Assist Tomogr 16:254-260. https://doi.org/10.1097/00004728-19920300000014

27. Kreuz PC, Steinwachs MR, Erggelet $C$ et al (2006) Results after microfracture of full-thickness chondral defects in different compartments in the knee. Osteoarthritis Cartilage 14:1119-1125. https://doi.org/10.1016/j.joca.2006.05.003

28. Lu Y, Li YJ, Guo SY, Zhang HL (2018) Is there any difference between open and arthroscopic treatment for osteochondritis dissecans (OCD) of the humeral capitellum: a systematic review and meta-analysis. Int Orthop 42:601-607. https://doi. org/10.1007/s00264-018-3768-3

29. Makino T, Fujioka H, Kurosaka M et al (2001) Histologic analysis of the implanted cartilage in an exact-fit osteochondral transplantation model. Arthrosc J Arthrosc Relat Surg 17:747-751. https:// doi.org/10.1053/jars.2001.24705

30. Maruyama M, Takahara M, Harada M et al (2014) Outcomes of an open autologous osteochondral plug graft for capitellar osteochondritis dissecans: time to return to sports. Am J Sports Med 42:2122-2127. https://doi.org/10.1177/ 0363546514538759

31. Maruyama M, Takahara M, Satake H (2018) Diagnosis and treatment of osteochondritis dissecans of the humeral capitellum. J Orthop Sc 23:213-219. https://doi.org/10.1016/j.jos.2017. 11.013

32. Matsuura T, Kashiwaguchi S, Iwase T et al (2008) Conservative treatment for osteochondrosis of the humeral capitellum. Am J Sports Med 36:868-872. https://doi.org/10.1177/0363546507312168

33. Mihara K, Tsutsui H, Nishinaka N, Yamaguchi K (2009) Nonoperative treatment for osteochondritis dissecans of the capitellum. Am J Sports Med 37:298-304. https://doi.org/10.1177/ 0363546508324970

34. Mubarak SJ, Carroll NC (1981) Juvenile osteochondritis dissecans of the knee: etiology. Clin Orthop Relat Res Jun(157):200-211.PMID:7018781

35. Niemeyer P, Albrecht D, Andereya S et al (2016) Autologous chondrocyte implantation $(\mathrm{ACl})$ for cartilage defects of the knee: a guideline by the working group "Clinical Tissue Regeneration" of the German Society of Orthopaedics and Trauma (DGOU). Knee 23:426-435. https://doi.org/10. 1016/j.knee.2016.02.001

36. Niu EL, Tepolt FA, Bae DS et al (2018) Nonoperative management of stable pediatric osteochondritis dissecans of the capitellum: predictors of treatment success. J Shoulder Elbow Surg 27:2030-2037. https://doi.org/10.1016/j.jse.2018. 07.017

37. PatzerT, KrauspeR,HufelandM(2016) Arthroscopic autologous chondrocyte transplantation for osteochondritis dissecans of the elbow. Arthrosc Tech 5:e633-e636. https://doi.org/10.1016/j.eats. 2016.02.019

38. Peterson L et al (1984) Chondrocyte transplantation. An experimental model in the rabbit. Trans Orthop Res Soc 30:218

39. Rosenberg ZS, Beltran J, Cheung YY (1994) Pseudodefect of the capitellum: potential MR imaging pitfall. Radiology 191:821-823. https:// doi.org/10.1148/radiology.191.3.8184072

40. Ruch DS, Cory JW, Poehling GG (1998) The arthroscopic management of osteochondritis dissecans of the adolescent elbow. Arthrosc J Arthrosc Relat Surg 14:797-803. https://doi.org/ 10.1016/s0749-8063(98)70013-1
41. Sato K, Iwamoto T, Matsumura N et al (2018) Costal osteochondral autograft for advanced osteochondritis dissecans of the humeral capitellum in adolescent and young adult athletes: clinical outcomes with a mean follow-up of 4.8 years. J Bone Joint Surg Am 100:903-913. https://doi. org/10.2106/JBJS.17.01035

42. Sato M, Ochi M, Uchio Yetal (2004) Transplantation of tissue-engineered cartilage for excessive osteochondritis dissecans of the elbow. J Shoulder Elbow Surg 13:221-225. https://doi.org/10.1016/j. jse.2003.09.012

43. Sauvegrain J, Nahum H, Bronstein H (1962) Study of bone maturation of the elbow. Ann Radiol (Paris) 5:542-550. (French. PMID:13986863)

44. Schenck RC, Goodnight JM (1996) Osteochondritis dissecans. J Bone Joint Surg Am 78:439-456

45. Shimada K, Tanaka H, Matsumoto T et al (2012) Cylindrical costal osteochondral autograft for reconstruction of large defects of the capitellum due to osteochondritis dissecans. JBone Joint Surg Am 94:992-1002. https://doi.org/10.2106/JBJS.J. 00228

46. Steadman JR, Rodkey WG, Rodrigo JJ (2001) Microfracture: surgical technique and rehabilitation to treat chondral defects. Clin Orthop. https://doi. org/10.1097/00003086-200110001-00033

47. Takahara M, Shundo M, Kondo M et al (1998) Early detection of osteochondritis dissecans of the capitellum in young baseball players. Report of three cases. J Bone Joint Surg Am 80:892-897. https://doi.org/10.2106/00004623-19980600000014

48. Takahara M, Ogino T, Fukushima S et al (1999) Nonoperative treatment of osteochondritis dissecans of the humeral capitellum. Am J Sports Med 27:728-732. https://doi.org/10.1177/ 03635465990270060701

49. Takahara M, Mura N, Sasaki J et al (2008) Classification, treatment, and outcome of osteochondritis dissecans of the humeral capitellum. Surgical technique. J Bone Joint Surg Am 90(Suppl 2 Pt 1):47-62.https://doi.org/10.2106/JBJS.G.01135

50. Thiele K, Hollinger B, Nietschke R et al (2019) Osteochondrale Läsionen am Ellbogen. Arthroskopie 32:269-278. https://doi.org/10.1007/s00142019-0288-0

51. Tsuda E, Ishibashi Y, Sato H et al (2005) Osteochondral autograft transplantation for osteochondritis dissecans of the capitellum in nonthrowing athletes. Arthrosc J Arthrosc Relat Surg 21:1270. https:// doi.org/10.1016/j.arthro.2005.06.006

52. Ueda Y, Sugaya H, Takahashi N et al (2017) Arthroscopic fragment resection for capitellar osteochondritis dissecans in adolescent athletes: 5- to 12-year follow-up. Orthop J Sports Med. https://doi.org/10.1177/2325967117744537

53. Versteylen RJ, Zwemmer A, Lorié CA, Schuur KH (1988) Multiple epiphyseal dysplasia complicated by severe osteochondritis dissecans of the knee. Incidence in two families. Skelet Radiol 17:407-412. https://doi.org/10.1007/bf00361659

54. Vogt S, Siebenlist S, Hensler D et al (2011) Osteochondral transplantation in the elbow leads to good clinical and radiologic long-term results: an 8- to 14-year follow-up examination. Am J Sports Med 39:2619-2625. https://doi.org/10. $1177 / 0363546511420127$

55. Waldt S, Bruegel M, Ganter K et al (2005) Comparison of multislice CT arthrography and MR arthrographyfor the detection of articularcartilage lesions of the elbow. Eur Radiol 15:784-791. https://doi.org/10.1007/s00330-004-2585-9 
56. Yamamoto Y, Ishibashi Y, Tsuda E et al (2006) Osteochondral autograft transplantation for osteochondritis dissecans of the elbow in juvenile baseball players: minimum 2-year follow-up. Am J Sports Med 34:714-720. https://doi.org/10.1177/ 0363546505282620

57. Yoshizuka $M$, Sunagawa $T$, Nakashima $Y$ et al (2018) Comparison of sonography and MRI in the evaluation of stability of capitellar osteochondritis dissecans. J Clin Ultrasound 46:247-252. https:// doi.org/10.1002/jcu.22563 